\title{
Isatuximab plus carfilzomib and dexamethasone versus carfilzomib and dexamethasone in relapsed multiple myeloma patients with renal impairment: IKEMA subgroup analysis
}

\begin{abstract}
Marcelo Capra, ${ }^{1}$ Thomas Martin, ${ }^{2}$ Philippe Moreau, ${ }^{3}$ Ross Baker, ${ }^{4}$ Ludek Pour, ${ }^{5}$ Chang-Ki Min, ${ }^{6}$ Xavier Leleu, ${ }^{7}$ Mohamad Mohty, ${ }^{8}$ Marta Reinoso Segura, ${ }^{9}$ Mehmet Turgut, ${ }^{10}$ Richard LeBlanc, ${ }^{11}$ Marie-Laure Risse, ${ }^{12}$ Laure Malinge, ${ }^{13}$ Sandrine Schwab ${ }^{12}$ and Meletios Dimopoulos ${ }^{14}$

${ }^{1}$ Centro Integrado de Hematologia e Oncologia, Hospital Mãe de Deus, Porto Alegre, Brazil; ${ }^{2}$ Department of Medicine, University of California at San Francisco, San Francisco, CA, USA; ${ }^{3}$ Department of Hematology, University of Nantes, Nantes, France; ${ }^{4}$ Perth Blood Institute, Murdoch University, Perth, Western Australia, Australia; ${ }^{5}$ Department of Internal Medicine, Hematology and Oncology, University Hospital Brno, Brno, Czech Republic; ${ }^{6}$ Department of Hematology, Catholic Hematology Hospital and Leukemia Research Institute, Seoul St. Mary's Hospital, College of Medicine, The Catholic University of Korea, Seoul, Korea; ${ }^{7}$ Service d'Hématologie et Thérapie Cellulaire, CHU and CIC INSERM 1402, Poitiers Cedex, France; ${ }^{8}$ Department of Hematology, Hôpital Saint-Antoine, Sorbonne University, INSERM UMRS 938, Paris, France; ${ }^{3}$ Hospital Universitario Virgen del Rocio, Sevilla, Spain; ${ }^{10}$ Department of Hematology, Ondokuz Mayıs University Faculty of Medicine, Samsun, Turkey; ${ }^{11}$ Hôpital Maisonneuve-Rosemont, Université de Montréal, Montréal, Quebec, Canada; ${ }^{12}$ Sanofi Research and Development, Vitry/Alfortville, France; ${ }^{13} \mathrm{Aixial}$, BoulogneBillancourt, France and ${ }^{14}$ Department of Clinical Therapeutics, School of Medicine, National and Kapodistrian University of Athens School of Medicine, Athens, Greece.
\end{abstract}

\author{
Correspondence: \\ Marcelo Capra \\ marcelocapra@hotmail.com \\ Received: May 28, 2021. \\ Accepted: $\quad$ October 5, 2021. \\ Prepublished: October 14, 2021. \\ https://doi.org/10.3324/haematol.2021.279229 \\ ๑2022 Ferrata Storti Foundation \\ Haematologica material is published under a CC \\ BY-NC license @( $) \Theta$
}

\begin{abstract}
Renal impairment (RI) is common in patients with multiple myeloma (MM) and new therapies that can improve renal function are needed. The phase III IKEMA study (clinicaltrials gov. Identifier: NCT03275285) investigated isatuximab (Isa) with carfilzomib and dexamethasone $(\mathrm{Kd})$ versus $\mathrm{Kd}$ in relapsed MM. This subgroup analysis examined results from patients with $\mathrm{Rl}$, defined as estimated glomerular filtration rate $<60 \mathrm{~mL} / \mathrm{min} / 1.73 \mathrm{~m}^{2}$. Addition of Isa prolonged progression-free survival (PFS) in patients with RI (hazard ratio: 0.27; 95\% confidence interval [CI]: 0.11-0.66; median PFS not reached for Isa-Kd versus 13.4 months for Kd [20.8-month follow-up]). Complete renal responses occurred more frequently with Isa-Kd (52.0\%) versus $\mathrm{Kd}(30.8 \%)$ and were durable in $32.0 \%$ versus $7.7 \%$ of patients, respectively. Treatment exposure was longer with Isa-Kd, with median number of started cycles and median duration of exposure of 20 versus 9 cycles and 81.0 versus 35.7 weeks for Isa-Kd versus Kd, respectively. Among patients with RI, the incidence of patients with grade $\geq 3$ treatment-emergent adverse events was similar between the two arms ( $79.1 \%$ in Isa-Kd vs. $77.8 \%$ in $\mathrm{Kd}$ ). In summary, the addition of Isa to Kd improved clinical outcomes with a manageable safety profile in patients with RI, consistent with the benefit observed in the overall IKEMA study population.
\end{abstract}

\section{Introduction}

Multiple myeloma (MM) is characterized by abnormal proliferation of plasma cells and production of M-protein, a monoclonal immunoglobulin (Ig). Renal impairment (RI) affects up to $50 \%$ of MM patients, depending on how RI is defined. MM-related RI is multifactorial, but mainly caused by precipitation of Ig-free light chains in the distal tubules, leading to tubule obstruction and cast nephropathy. ${ }^{1} \mathrm{RI}$ is a major cause of morbidity and an adverse predictor of survival in MM patients., ${ }^{2,3}$ As renal function recovery is associated with improved clinical outcomes, it is one of the main therapeutic goals in MM patients with RI. Urgent therapy is required to achieve reversal of severe RI, since renal failure established for $>2$ weeks would substantially compromise the possibility of recovery. ${ }^{4-7}$

Newly introduced anti-myeloma therapies such as proteasome inhibitors (i.e., bortezomib, carfilzomib) ${ }^{3,8-12}$ and immunomodulatory drugs (i.e., lenalidomide, pomalidomide) ${ }^{13-20}$ aid in the recovery of renal function. ${ }^{21,22}$ Carfilzomib is a nextgeneration proteasome inhibitor approved as monotherapy 
or in combination with dexamethasone (Kd), lenalidomide/ dexamethasone, or daratumumab/dexamethasone for relapsed/refractory MM (RRMM). ${ }^{8,23}$ The phase III ENDEAVOR study demonstrated superiority of Kd versus bortezomib/ dexamethasone $(\mathrm{Vd})$ in RRMM patients with 1-3 prior treatment lines. ${ }^{8}$ Median progression-free survival (PFS) was 18.7 months with $\mathrm{Kd}$ versus 9.4 months with $\mathrm{Vd}$ (hazard ratio [HR]: 0.53; 95\% confidence interval [Cl]: 0.44-0.65; $P<0.0001]$. Median overall survival (OS) was 47.6 months with Kd versus 40.0 months with Vd (HR: 0.791; 95\% Cl: 0.65-0.96; one-sided $P=0.010$ ). A post-hoc exploratory subgroup analysis of ENDEAVOR reported complete renal response in 15.3\% of Kd-treated patients, with longer median PFS and OS in patients achieving complete renal responses. ${ }^{9}$ The ENDEAVOR study results showed activity in patients with renal function impairment, supporting $\mathrm{Kd}$ as a therapeutic option for MM patients with RI. ${ }^{7-9}$ However, Kd treatment in patients with RI may present challenges, as carfilzomib has been associated with renal toxicity and hypertension, and may require repeated administration of intravenous fluids compared with oral or subcutaneous alternatives. ${ }^{7-9,23}$

Based on the phase III ICARIA-MM study, isatuximab (Isa), an anti-CD38 monoclonal antibody, is approved in a number of countries in combination with pomalidomide/ dexamethasone for the treatment of RRMM patients who have received $\geq 2$ prior therapies, including lenalidomide and a proteasome inhibitor. ${ }^{24-27}$ Based on the phase III IKEMA study, Isa to date is also approved in combination with $\mathrm{Kd}$ in the United States for patients with relapsed MM who have received 1-3 prior treatment lines and in the European Union for MM patients who have received $\geq 1$ prior therapy. ${ }^{24,25,28}$

A pre-specified IKEMA interim analysis showed that PFS was prolonged by the addition of Isa (median PFS, not reached for Isa-Kd versus 19.2 months with Kd; stratified HR: 0.53; 99\% Cl: 0.32-0.89; one-sided log-rank test $P=0.0007)$, crossing the pre-specified efficacy boundary $(P=0.005){ }^{28}$ This pre-specified subgroup analysis of IKEMA examined efficacy, renal response, and safety in patients with RI, at the time of the interim analysis.

\section{Methods}

\section{Study design}

IKEMA (clinicaltrials gov. Identifier: NCT03275285) was a prospective, multinational, randomized, open-label, parallel-group, phase III study conducted at 69 study centers in 16 countries ${ }^{29}$ Institutional ethics committees or independent review boards approved the study protocol for each center. The study was conducted in accordance with the Declaration of Helsinki and the International Conference on Harmonization Guidelines for Good Clinical Practice. All patients provided written informed consent.

\section{Patients}

Details of the study methodology have been reported previously. ${ }^{28,29}$ Briefly, eligible patients had relapsed MM with 1-3 prior lines of therapy. Patients were excluded if they had primary refractory MM or serum free-light chain measurable disease only, had received prior carfilzomib treatment, were refractory to anti-CD38 antibody therapy, or presented with left ventricular ejection fraction $<40 \%$. Patients with a baseline estimated glomerular filtration rate (eGFR) as low as $15 \mathrm{~mL} / \mathrm{min} / 1.73 \mathrm{~m}^{2}$ were eligible for enrolment. ${ }^{30}$ Patients with prior pulmonary comorbidities, including chronic obstructive pulmonary disease, could be enrolled. ${ }^{29}$

\section{Randomization}

Patients were randomly assigned in a 3:2 ratio to receive Isa-Kd or Kd. Randomization was stratified by number of prior treatment lines (1 vs. >1) and Revised International Staging System (R-ISS) stage I or II versus stage III versus not classified, at study entry. ${ }^{28}$

\section{Treatment}

Patients in the Isa-Kd arm received Isa intravenously at 10 $\mathrm{mg} / \mathrm{kg}$ on days $1,8,15$, and 22 in the first 28-day cycle; and days 1 and 15 in subsequent cycles. In both arms, carfilzomib was administered intravenously at $20 \mathrm{mg} / \mathrm{m}^{2}$ on days 1 and 2; $56 \mathrm{mg} / \mathrm{m}^{2}$ on days $8,9,15$, and 16 of cycle 1 ; and then $56 \mathrm{mg} / \mathrm{m}^{2}$ on days $1,2,8,9,15$, and 16 of subsequent cycles. ${ }^{28}$ Dexamethasone $20 \mathrm{mg}$ was administered intravenously or orally on days 1, 2, 8, 9, 15, 16, 22, and 23. Treatment continued until unacceptable adverse event ( $A E)$, disease progression, or other discontinuation criteria.

\section{Study endpoints and measured outcomes}

The primary efficacy endpoint was PFS, as per blinded independent response committee (IRC). The IRC reviewed disease assessments for response and progression (central radiological evaluation, $\mathrm{M}$-protein quantification from central laboratory, and local bone marrow aspiration for plasma cell infiltration when needed).

Key secondary efficacy endpoints included overall response rate (ORR) according to the International Myeloma Working Group (IMWG) response criteria, ${ }^{31}$ very good partial response (VGPR) or better rate, measurable residual disease (MRD) negativity rate, complete response (CR) rate, and OS. ${ }^{32-34} \mathrm{MRD}$ was assessed by central laboratory using next-generation sequencing (NGS) Adaptive clonoSEQ Assay (Adaptive Biotechnologies, Seattle, WA) with a minimum sensitivity of $1 / 10^{5}$ nucleated cells in patients reaching $\geq V G P R$.

Efficacy assessments were performed on day 1 of every cycle and at end of treatment. Safety assessments included recording of AE (graded per NCI-CTCAE v4.03), lab- 
oratory parameters (including complete blood, neutrophil, and platelet counts; and hemoglobin values, graded per $\mathrm{NCl}$-CTCAE version 4.03), vital signs, electrocardiograms, and Eastern Cooperative Oncology Group performance status (ECOG PS). Safety was regularly reviewed by an Independent Data Monitoring Committee.

\section{Renal response}

Both renal function impairment and renal response were analyzed. The eGFR was assessed using the modification of diet in renal disease (MDRD) equation on days 1, 2, 8, 9, 15,16 , and 22 of cycle 1 ; days 1, 8, and 15 of cycle 2 ; days 1 and 15 of each subsequent treatment cycle, and as clinically indicated. eGFR results were classified as RI $(<60$ $\left.\mathrm{mL} / \mathrm{min} / 1.73 \mathrm{~m}^{2}\right)$ or no RI $\left(\geq 60 \mathrm{~mL} / \mathrm{min} / 1.73 \mathrm{~m}^{2}\right)$. Based on IMWG criteria, complete renal response was defined as an increase in eGFR from $<50 \mathrm{~mL} / \mathrm{min} / 1.73 \mathrm{~m}^{2}$ at baseline to $\geq 60 \mathrm{~mL} / \mathrm{min} / 1.73 \mathrm{~m}^{2}$ (no RI) in $\geq 1$ post-baseline assessment. ${ }^{6,7}$ Responses were considered durable when lasting $\geq 60$ days. ${ }^{6} \mathrm{~A}$ minor renal response was defined as an improvement in eGFR from $\geq 15$ to $<30 \mathrm{~mL} / \mathrm{min} / 1.73 \mathrm{~m}^{2}$ at baseline to $\geq 30 \mathrm{~mL} / \mathrm{min} / 1.73 \mathrm{~m}^{2}$ in $\geq 1$ assessment during treatment. $^{7}$

\section{Statistical analysis}

Sample size calculation was based on the primary efficacy endpoint; 159 events were needed to detect a $41 \%$ lower risk of disease progression (HR: 0.59) using a log-rank test (one-sided significance level of $0.025,90 \%$ power). An interim PFS analysis was pre-planned when $65 \%$ of the 159 PFS events (103 events) were observed to detect overwhelming efficacy.

All efficacy analyses were conducted in the intent-to-treat population and summarized by randomized treatment. Extent of study treatment and treatment-emergent $\mathrm{AE}$ (TEAE) analyses were conducted in the safety population. Median PFS, probabilities of being progression-free, and corresponding $\mathrm{Cl}$ were estimated using the Kaplan-Meier method. HR estimates were determined using the Cox proportional-hazard model by subgroup. Comparisons between patients with and without RI were observational only, with no formal statistical analysis performed. SAS 9.4 (SAS, Cary, NC) was used for all analyses.

\section{Results}

\section{Patients}

A total of 302 patients were randomized to Isa-Kd $(n=179)$ or $K d(n=123)$. Baseline eGFR values could be calculated for 165 patients in the Isa-Kd arm and 111 in the Kd arm. Baseline eGFR was not evaluable for 14 patients in Isa-Kd and 12 in $\mathrm{Kd}$, due to local legal restrictions on collecting racial group information. Among evaluable patients, 43
(26.1\%) in the Isa-Kd arm and 18 (16.2\%) in the Kd arm had RI (eGFR $<60 \mathrm{~mL} / \mathrm{min} / 1.73 \mathrm{~m}^{2}$ ). Of these, 39 (23.6\%) patients in Isa-Kd and 15 (13.5\%) in $\mathrm{Kd}$ had moderate RI (eGFR $\geq 30$ to $<60 \mathrm{~mL} / \mathrm{min} / 1.73 \mathrm{~m}^{2}$ ); $2.4 \%$ of patients in Isa$\mathrm{Kd}$ and $2.7 \%$ in $\mathrm{Kd}$ had severe $\mathrm{RI}$ (eGFR $\geq 15$ to $<30$ $\mathrm{mL} / \mathrm{min} / 1.73 \mathrm{~m}^{2}$ ).

Among patients with $\mathrm{Rl}$ at baseline, characteristics were generally well balanced between study arms (Table 1), except for more patients aged $\geq 75$ years in the Isa-Kd than the $\mathrm{Kd}$ arm (14.0\% vs. 5.6\%, respectively) and more patients refractory to lenalidomide $(25.6 \%$ Isa-Kd vs. $50.0 \% \mathrm{Kd}$ ) or to immunomodulatory drugs and proteasome inhibitors $(18.6 \%$ Isa-Kd vs. $44.4 \% \mathrm{Kd})$ in the control arm. Patients with $\mathrm{RI}$ in both the Isa-Kd and $\mathrm{Kd}$ arms tended to be older, had more ISS stage III disease, and received more prior therapy lines than patients without RI (Table 1). Patient flow was described previously. ${ }^{28}$

\section{Efficacy}

At a median overall follow-up of 20.7 months, the PFS benefit of Isa-Kd versus $\mathrm{Kd}$ in patients with and without $\mathrm{RI}$, according to the assessment by the IRC, was consistent with that seen for the overall IKEMA study population (Figure 1). The addition of Isa prolonged PFS in patients with RI (HR: 0.27; 95\% Cl: 0.11-0.66; median PFS not reached for Isa-Kd vs. 13.4 months for Kd) and in patients without RI (HR: 0.63; 95\% Cl: 0.39-1.00; medians not reached). Probability to be free of a PFS event at 18 months was $79 \%$ with Isa-Kd versus $41 \%$ with $\mathrm{Kd}$ in patients with $\mathrm{RI}$ and $71 \%$ with Isa-Kd versus $59 \%$ with $\mathrm{Kd}$ in those without RI.

Multivariate analysis of PFS for patients with RI was performed to adjust the imbalance at baseline between Isa$\mathrm{Kd}$ and $\mathrm{Kd}$, including ISS stage, gain(1q21), refractory to PI or IMiD therapy, sex, and regulatory region as covariates. Adjusted HR was equal to 0.21 (95\% Cl: $0.07-0.68$ ), suggesting that the imbalance did not influence the treatment effect in favor of Isa-Kd for PFS.

Consistent treatment effect was also observed in patients with the most severe RI at baseline (eGFR $<45$ $\mathrm{mL} / \mathrm{min} / 1.73 \mathrm{~m}^{2}$ ), as an exploratory analysis, in favor of patients treated in Isa-Kd (HR: 0.16 ; 95\% Cl: 0.04-0.67; median PFS, not reached for Isa-Kd $[n=19]$ versus 11.14 months for $K d[n=10])$ and in patients with eGFR $\geq 45$ $\mathrm{mL} / \mathrm{min} / 1.73 \mathrm{~m}^{2}$ (HR: 0.60; 95\% Cl: 0.39-0.93; medians not reached, $n=146$ versus $n=101$ ).

In the intent-to-treat population, the ORR was higher with Isa-Kd versus $\mathrm{Kd}$ for patients with RI $(93.1 \%$ vs. $61.1 \%$, respectively; Figure 2). Although the ORR was $83.6 \%$ with Isa$\mathrm{Kd}$ versus $89.2 \%$ with $\mathrm{Kd}$ for patients without $\mathrm{RI}$, the $\geq \mathrm{VGPR}$ rate for patients with RI was $79.1 \%$ with Isa-Kd versus $44.4 \%$ with $\mathrm{Kd}$, and for patients without $\mathrm{RI}$, it was $71.3 \%$ versus $59.1 \%$, respectively. The MRD negativity rate, assessed by NGS at $10^{-5}$ sensitivity level in bone marrow aspirates from 
Table 1. Baseline characteristics in patients with and without renal impairment in the isatuximab (Isa) carfilzomib (K) dexamethasone (d) (Isa-Kd) and Kd arms - intent-to-treat population

\begin{tabular}{|c|c|c|c|c|}
\hline & \multicolumn{2}{|c|}{ eGFR $<60 \mathrm{~mL} / \mathrm{min} / 1.73 \mathrm{~m}^{2}(\mathrm{n}=61)$} & \multicolumn{2}{|c|}{ eGFR $\geq 60 \mathrm{~mL} / \mathrm{min} / 1.73 \mathrm{~m}^{2}(\mathrm{n}=215)$} \\
\hline & Isa-Kd ( $n=43)$ & Kd $(n=18)$ & Isa-Kd $(n=122)$ & $K d(n=93)$ \\
\hline Median age, years (range) & $67(39-86)$ & $69(49-90)$ & $64(37-81)$ & $62(33-78)$ \\
\hline \multicolumn{5}{|c|}{ Age in years by category, $\mathrm{n}(\%)$} \\
\hline$<65$ & $15(34.9)$ & $4(22.2)$ & $64(52.5)$ & $55(59.1)$ \\
\hline $65-74$ & $22(51.2)$ & $13(72.2)$ & $47(38.5)$ & $31(33.3)$ \\
\hline$\geq 75$ & $6(14.0)$ & $1(5.6)$ & $11(9.0)$ & $7(7.5)$ \\
\hline \multicolumn{5}{|c|}{ ISS stage at initial diagnosis, n (\%) } \\
\hline Stage I & $8(18.6)$ & $4(22.2)$ & $29(23.8)$ & $23(24.7)$ \\
\hline Stage II & $9(20.9)$ & $6(33.3)$ & $40(32.8)$ & $39(41.9)$ \\
\hline Stage III & $18(41.9)$ & $7(38.9)$ & $30(24.6)$ & $16(17.2)$ \\
\hline Unknown & $8(18.6)$ & $1(5.6)$ & $23(18.9)$ & $15(16.1)$ \\
\hline \multicolumn{5}{|c|}{ R-ISS stage at study entry, n (\%) } \\
\hline Stage I & $3(7.0)$ & $2(11.1)$ & $36(29.5)$ & $28(30.1)$ \\
\hline Stage II & $34(79.1)$ & $11(61.1)$ & $72(59.0)$ & $52(55.9)$ \\
\hline Stage III & $5(11.6)$ & $3(16.7)$ & $8(6.6)$ & $5(5.4)$ \\
\hline Not classified & $1(2.3)$ & $2(11.1)$ & $6(4.9)$ & $8(8.6)$ \\
\hline \multicolumn{5}{|l|}{ Cytogenetic riskª, n (\%) } \\
\hline High risk & $9(20.9)$ & $5(27.8)$ & $30(24.6)$ & $24(25.8)$ \\
\hline Standard risk & $29(67.4)$ & $12(66.7)$ & $76(62.3)$ & $57(61.3)$ \\
\hline Missing & $5(11.6)$ & $1(5.6)$ & $16(13.1)$ & $12(12.9)$ \\
\hline \multicolumn{5}{|l|}{ Prior lines of therapy } \\
\hline Median (range) & $2(1-4)$ & $2(1-4)$ & $2(1-3)$ & $1(1-4)$ \\
\hline 1 line, $n(\%)$ & $17(39.5)$ & $5(27.8)$ & $55(45.1)$ & $47(50.5)$ \\
\hline$\geq 2$ lines, n (\%) & $26(60.5)$ & $13(72.2)$ & $67(54.9)$ & $46(49.5)$ \\
\hline \multicolumn{5}{|l|}{ Patients refractory to, n (\%) } \\
\hline Lenalidomide & $11(25.6)$ & $9(50.0)$ & $39(32.0)$ & $26(28.0)$ \\
\hline IMiD and PI & $8(18.6)$ & $8(44.4)$ & $23(18.9)$ & $15(16.1)$ \\
\hline
\end{tabular}

${ }^{a}$ High risk was defined as del(17p), $\mathrm{t}(4 ; 14)$, or $\mathrm{t}(14 ; 16)$ by fluorescence in situ hybridization. Cytogenetics was assessed by a central laboratory with a cut-off of $50 \%$ for del(17p), and $30 \%$ for $\mathrm{t}(4 ; 14)$ and $\mathrm{t}(14 ; 16)$. d: dexamethasone; eGFR: estimated glomerular filtration rate; IMiD: immunomodulatory drug; Isa: isatuximab; ISS: International Staging System; ITT: intent-to-treat; K: carfilzomib; PI: proteasome inhibitor; RI: renal impairment; R-ISS: revised International Staging System.

patients who achieved $\geq$ VGPR, was $30.2 \%$ with Isa-Kd versus $11.1 \%$ with $\mathrm{Kd}$ for patients with RI and $29.5 \%$ with Isa$\mathrm{Kd}$ versus $14.0 \%$ with $\mathrm{Kd}$ for patients without RI. In addition, the CR rate for patients with RI was $41.9 \%$ with Isa-Kd versus $22.2 \%$ with $\mathrm{Kd}$, and for patients without RI, it was $40.2 \%$ versus $30.1 \%$, respectively (Figure 2 ).

Although OS data were not mature at the interim analysis, $17 \%$ and $20 \%$ of patients died in the Isa-Kd and $\mathrm{Kd}$ arms, respectively (among patients with $\mathrm{RI}: 12 \%$ in Isa-Kd versus $39 \%$ in $\mathrm{Kd}$ and among patients without RI: $18 \%$ in Isa-Kd versus $15 \%$ in $\mathrm{Kd}$ ).

\section{Renal response}

Among the 25 and 13 patients in the Isa-Kd and Kd arms, respectively, with eGFR $<50 \mathrm{~mL} / \mathrm{min} / 1.73 \mathrm{~m}^{2}$ at baseline, more patients in the Isa-Kd than the $\mathrm{Kd}$ arm had a complete renal response (52.0\% vs. 30.8\%; Figure $3 \mathrm{~A})$. Durable complete renal response occurred in eight of 25 (32.0\%) Isa-Kd versus one of $13(7.7 \%) \mathrm{Kd}$ patients. In patients with severe RI at baseline (eGFR $\geq 15$ to $<30 \mathrm{~mL} / \mathrm{min} / 1.73 \mathrm{~m}^{2}$ ), all patients in the Isa-Kd arm achieved minor renal response compared with only one patient in the $\mathrm{Kd}$ arm (4/4 [100\%] versus $1 / 3$ [33.3\%], respectively) (Figure 3A). Moreover, the time to first renal response and time to complete renal response were shorter in patients with baseline eGFR $<50 \mathrm{~mL} / \mathrm{min} / 1.73 \mathrm{~m}^{2}$ treated with Isa-Kd. Median time $(95 \% \mathrm{Cl})$ to first renal response was 1.51 (0.82-not calculable [NC]) months in the Isa-Kd arm ver- 
sus $6.51(0.69-\mathrm{NC})$ months in the $\mathrm{Kd}$ arm (Figure 3B). Median time $(95 \% \mathrm{Cl})$ to complete renal response was 7.82 (1.22-NC) months in the Isa-Kd arm versus NC (1.28-NC) months in the $\mathrm{Kd}$ arm (Figure $3 \mathrm{C}$ ).

A similar incidence of patients experienced, at least once, end-stage RI (eGFR $<15 \mathrm{~mL} / \mathrm{min} / 1.73 \mathrm{~m}^{2}$ ) during treatment with Isa-Kd versus $\mathrm{Kd}(1.8 \%$ vs. $2.7 \%$, respectively). In the safety population, the number of patients with $\geq 1 \mathrm{TEAE}$ mapped in the acute renal failure Standardized MedDRA
Queries (SMQ) narrow terms was nine of 177 (5.1\%, of which $1.1 \%$ were grade $\geq 3$ ) in the Isa-Kd arm versus ten of 122 (8.2\%, $2.5 \%$ grade $\geq 3)$. Acute kidney injury was observed in five of $177(2.8 \%, 1.1 \%$ grade $\geq 3)$ Isa-Kd patients versus seven of $122(5.7 \%, 1.6 \%$ grade $\geq 3) \mathrm{Kd}$ patients.

\section{Treatment exposure}

Longer treatment duration was observed with Isa-Kd versus $\mathrm{Kd}$ in patients with and without RI (Table 2). The

A

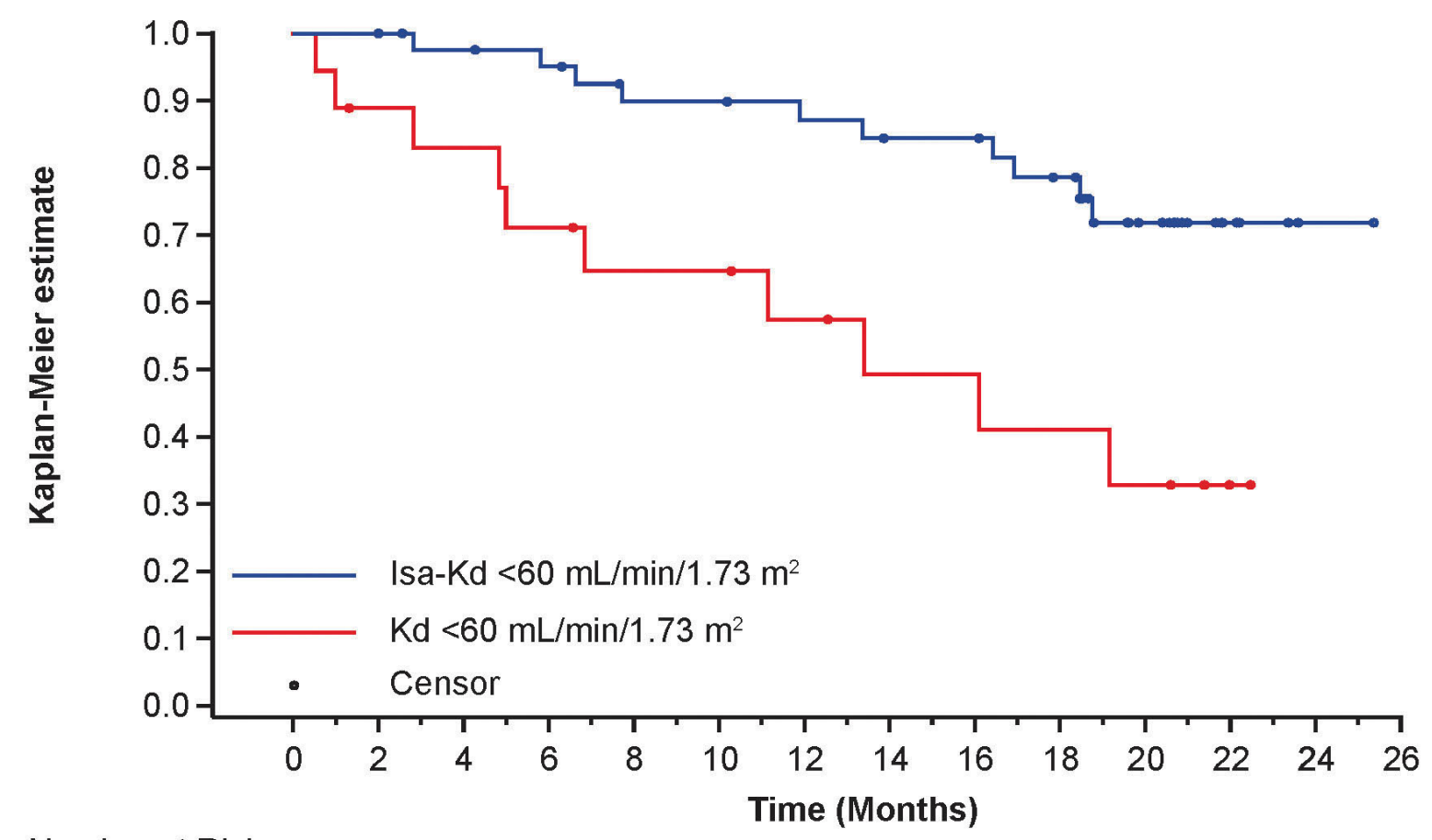

Number at Risk

Isa-Kd $<60 \mathrm{~mL} / \mathrm{min} / 1.73 \mathrm{~m}^{2} 43$

$\mathrm{Kd}<60 \mathrm{~mL} / \mathrm{min} / 1.73 \mathrm{~m}^{2} 18$

12

32

26

1

\section{B}

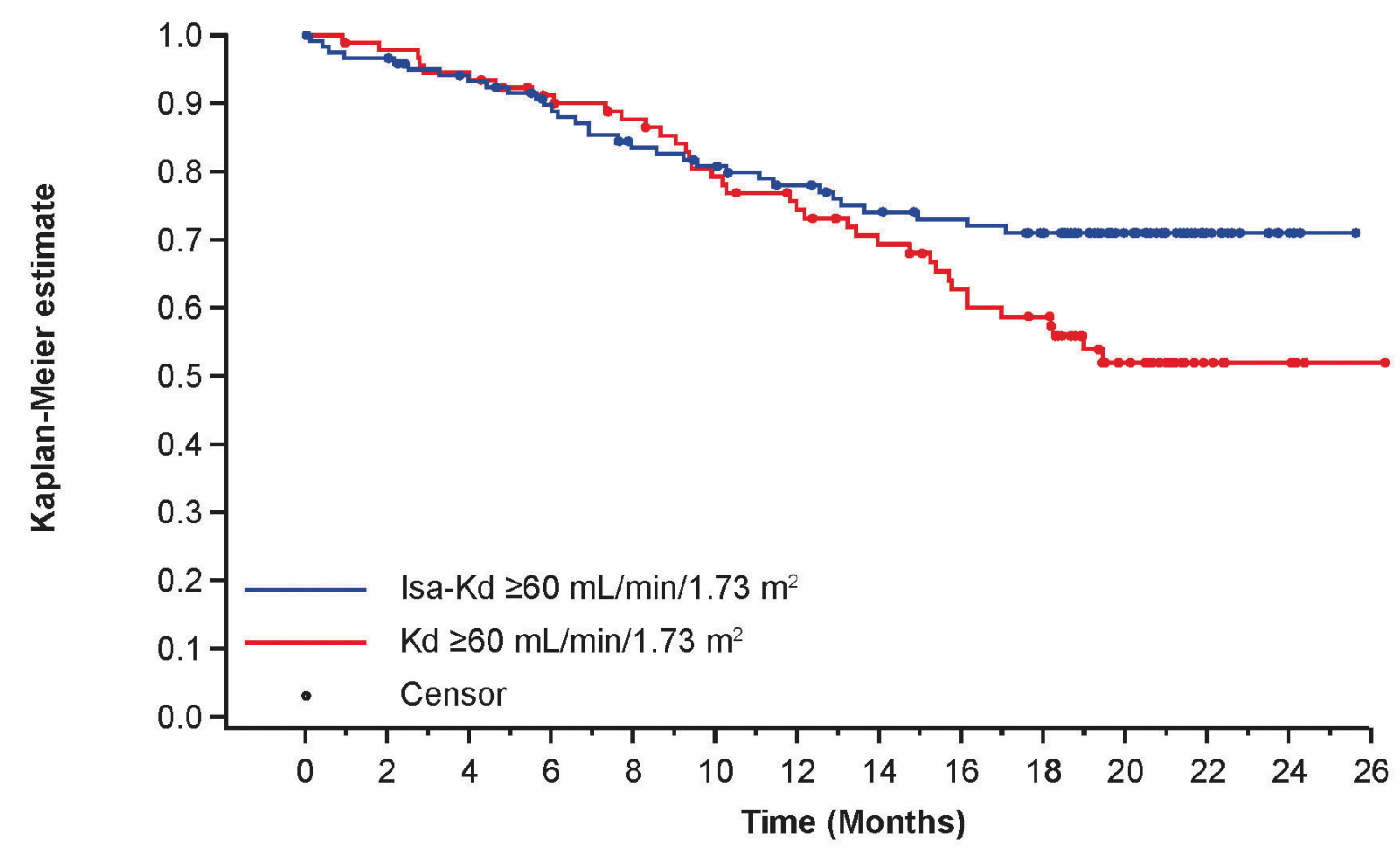

Number at Risk

Isa-Kd $\geq 60 \mathrm{~mL} / \mathrm{min} / 1.73 \mathrm{~m}^{2} 122$

101

79

82

67

4

$\mathrm{Kd} \geq 60 \mathrm{~mL} / \mathrm{min} / 1.73 \mathrm{~m}^{2} \quad 93$

60

43

6

Figure 1. Progression-free survival with isatuximab (Isa) carfilzomib (K) dexamethasone (d) (Isa-Kd) compared with Kd. (A) Patients with renal impairment (RI) (eGFR $<60 \mathrm{~mL} / \mathrm{min} / 1.73 \mathrm{~m}^{2}$ ) or (B) without RI (eGFR $\geq 60 \mathrm{~mL} / \mathrm{min} / 1.73 \mathrm{~m}{ }^{2}$ ), (ITT population). Progression-free survival (PFS) as per blinded independent response committee. d: dexamethasone; eGFR: estimated glomerular filtration rate; Isa: isatuximab; ITT: intent to treat; K: carfilzomib; PFS: progression-free survival. 
median (range) number of cycles for Isa-Kd was 20 (2-25) for patients with RI and 19 (1-27) cycles for those without $\mathrm{RI}$, and for $\mathrm{Kd}$ it was 9 (1-24) cycles for patients with RI and 17 (1-28) cycles for those without RI. Median duration of exposure for patients with and without RI was 81.0 (6104) and 78.6 (1-111) weeks with Isa-Kd versus 35.7 (1-97) and 68.5 (4-114) weeks with $\mathrm{Kd}$. More patients were still on treatment at the cut-off date in the Isa-Kd arm (55.8\% with $\mathrm{RI}$ and $54.1 \%$ without $\mathrm{RI}$ ) versus the $\mathrm{Kd}$ arm (16.7\% with $\mathrm{RI}$ and $36.6 \%$ without RI). The reasons for definitive treatment discontinuation in patients with RI were progressive disease $(27.9 \%$ in the Isa-Kd vs. $33.3 \%$ in the $\mathrm{Kd}$ arm) and $\mathrm{AE}(7.0 \%$ in the Isa-Kd vs. $27.8 \%$ in the $\mathrm{Kd}$ arm). In patients without RI, $26.2 \%$ in the Isa-Kd versus $37.6 \%$ in the $\mathrm{Kd}$ arm discontinued treatment due to progressive disease and $9.8 \%$ in the Isa-Kd versus $9.7 \%$ in the $\mathrm{Kd}$ arm due to $\mathrm{AE}$.
The median relative dose intensity of Isa was similar in patients with or without RI; thus, RI did not impact Isa administration. The relative dose intensity of carfilzomib in patients with RI was lower in the $\mathrm{Kd}$ arm (84.6\%) than in the Isa-Kd arm (93.1\%), but similar in patients without $\mathrm{RI}(90.1 \%$ in the Isa-Kd vs. $91.4 \%$ in the $\mathrm{Kd}$ arm), indicating that more carfilzomib doses were delayed, reduced, or omitted in patients with $\mathrm{RI}$ who received Kd (Table 2).

\section{Safety}

TEAE were experienced in $97.7 \%$ of Isa-Kd versus $100 \%$ of Kd patients with RI, whereas $93.7 \%$ versus $94.6 \%$ of patients without RI experienced TEAE in the Isa-Kd versus $\mathrm{Kd}$ arms, respectively (Table 3 ). In patients with $\mathrm{RI}$, grade $\geq 3$ TEAE were reported in $79.1 \%$ of Isa-Kd versus $77.8 \%$ of $\mathrm{Kd}$ patients and serious TEAE in $62.8 \%$ of Isa-Kd versus $77.8 \%$ of $\mathrm{Kd}$ pa-
A

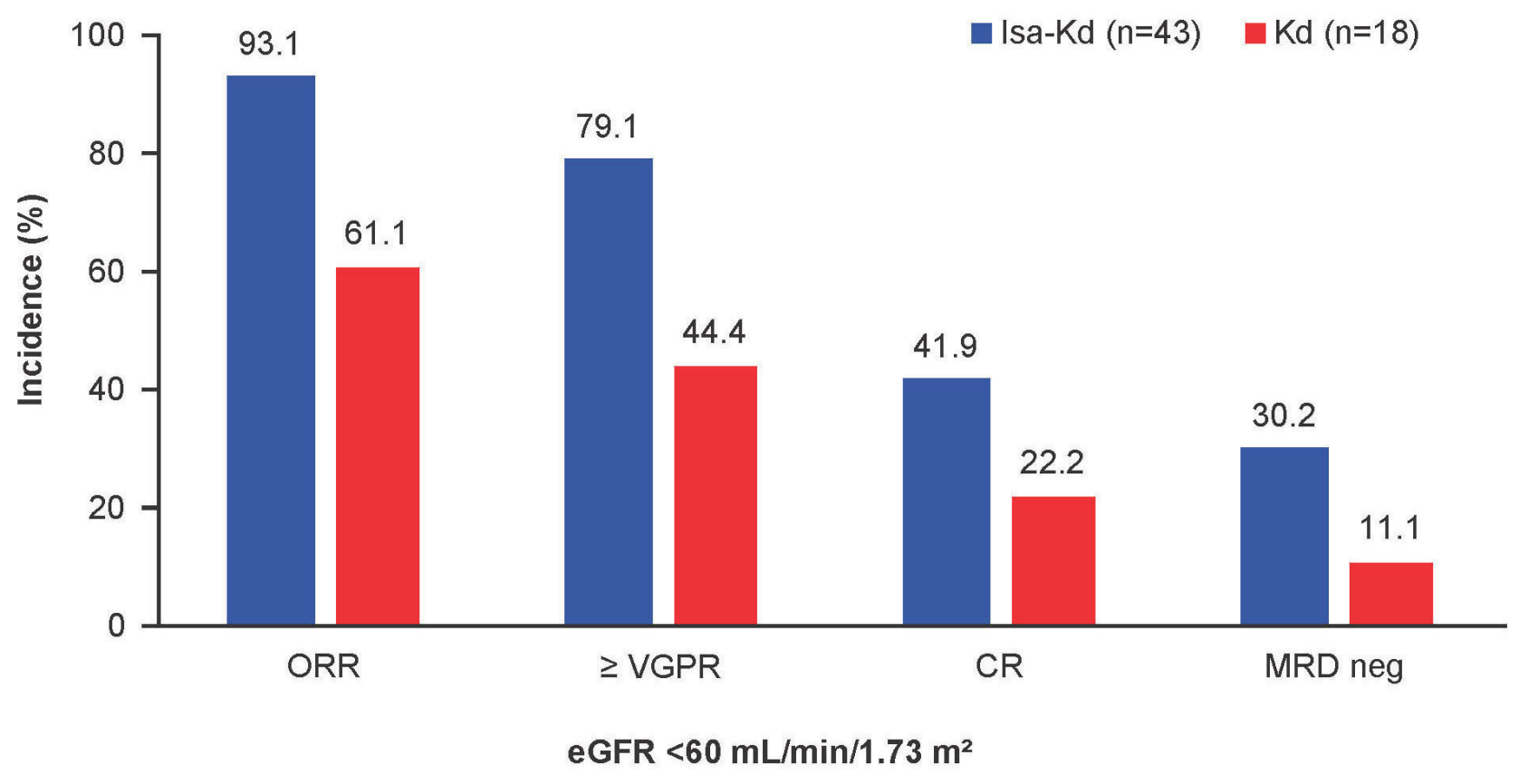

B

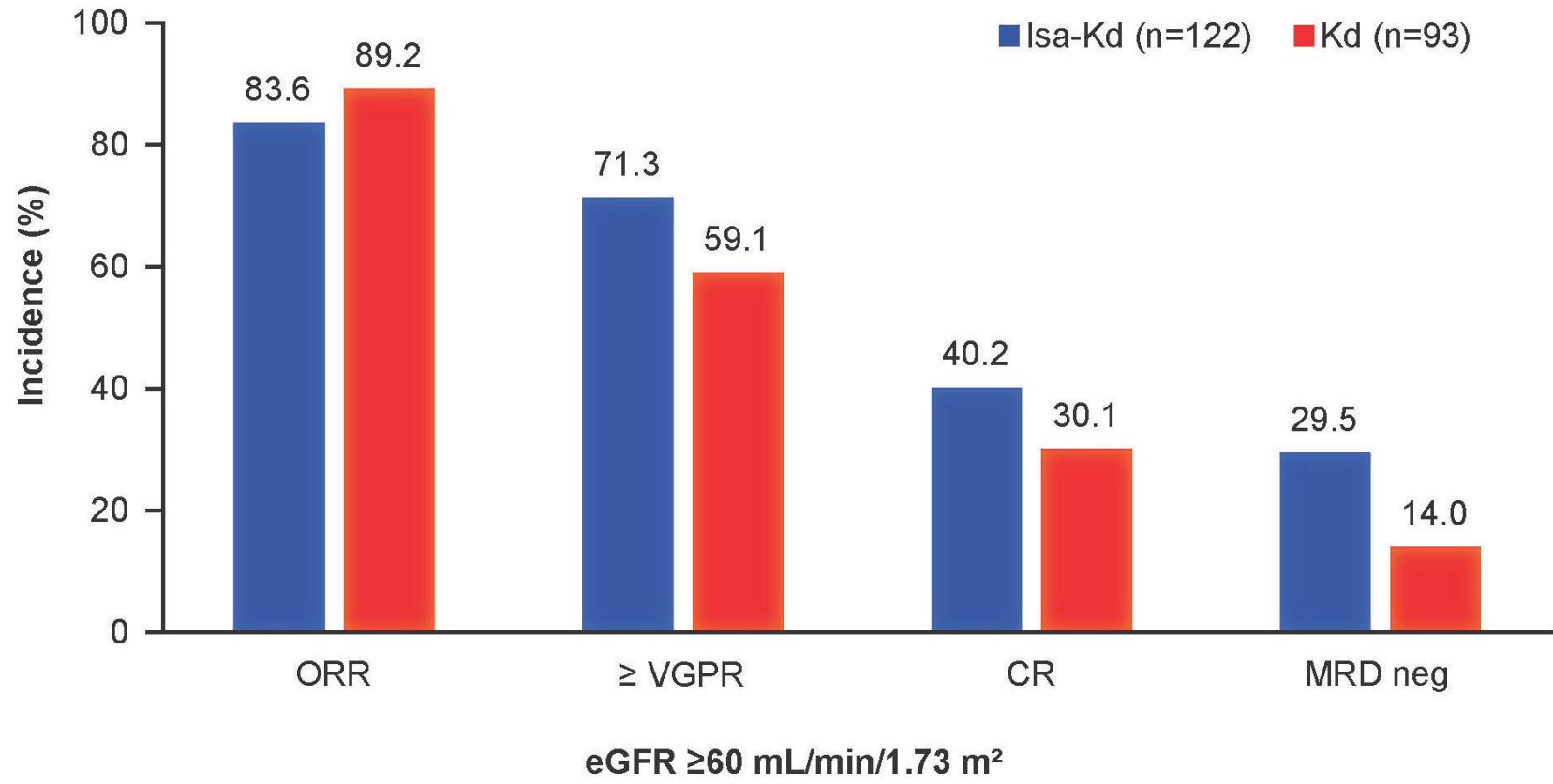

Figure 2. Response rates with isatuximab (Isa) carfilzomib (K) dexamethasone (d) (Isa-Kd) compared with Kd. (A) Patients with renal impairment (RI) (eGFR $<60 \mathrm{~mL} / \mathrm{min} / 1.73 \mathrm{~m}^{2}$ ) or (B) without RI (eGFR $\geq 60 \mathrm{~mL} / \mathrm{min} / 1.73 \mathrm{~m}^{2}$ ), (ITT population). CR: complete response; $d$ : dexamethasone; eGFR: estimated glomerular filtration rate; Isa: isatuximab; ITT: intent to treat; K: carfilzomib; MRD neg: minimal residual disease negativity; ORR: overall response rate; PR: partial response; VGPR: very good partial response. MRD was assessed by next-generation sequencing with a sensitivity level $10^{-5}$. 

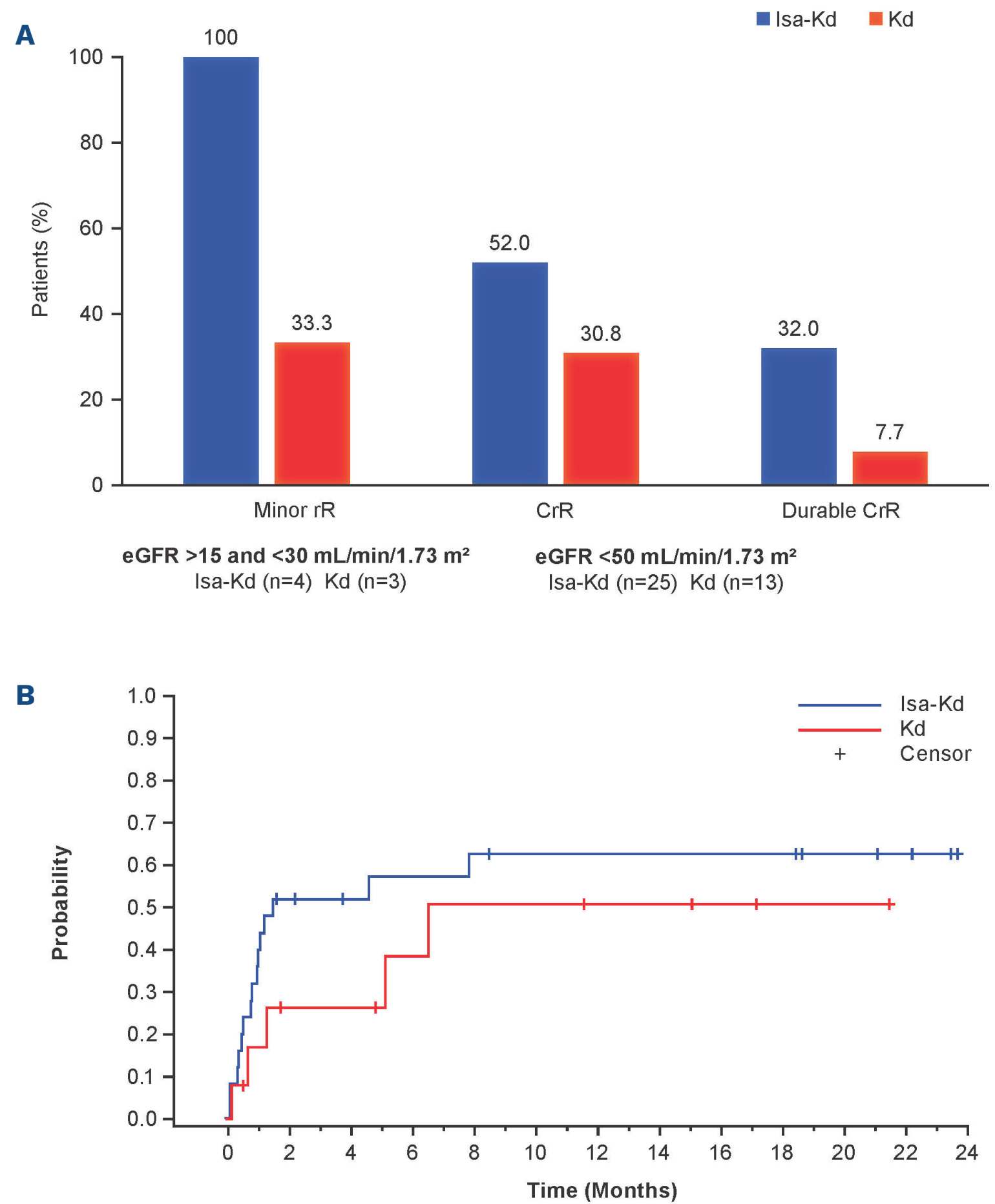

Number at Risk

$\begin{array}{rrrrrrr}\text { Isa-Kd } & 25 & 11 & 9 & 8 & 6 & 6 \\ \text { Kd } & 13 & 7 & 7 & 5 & 3 & 1\end{array}$

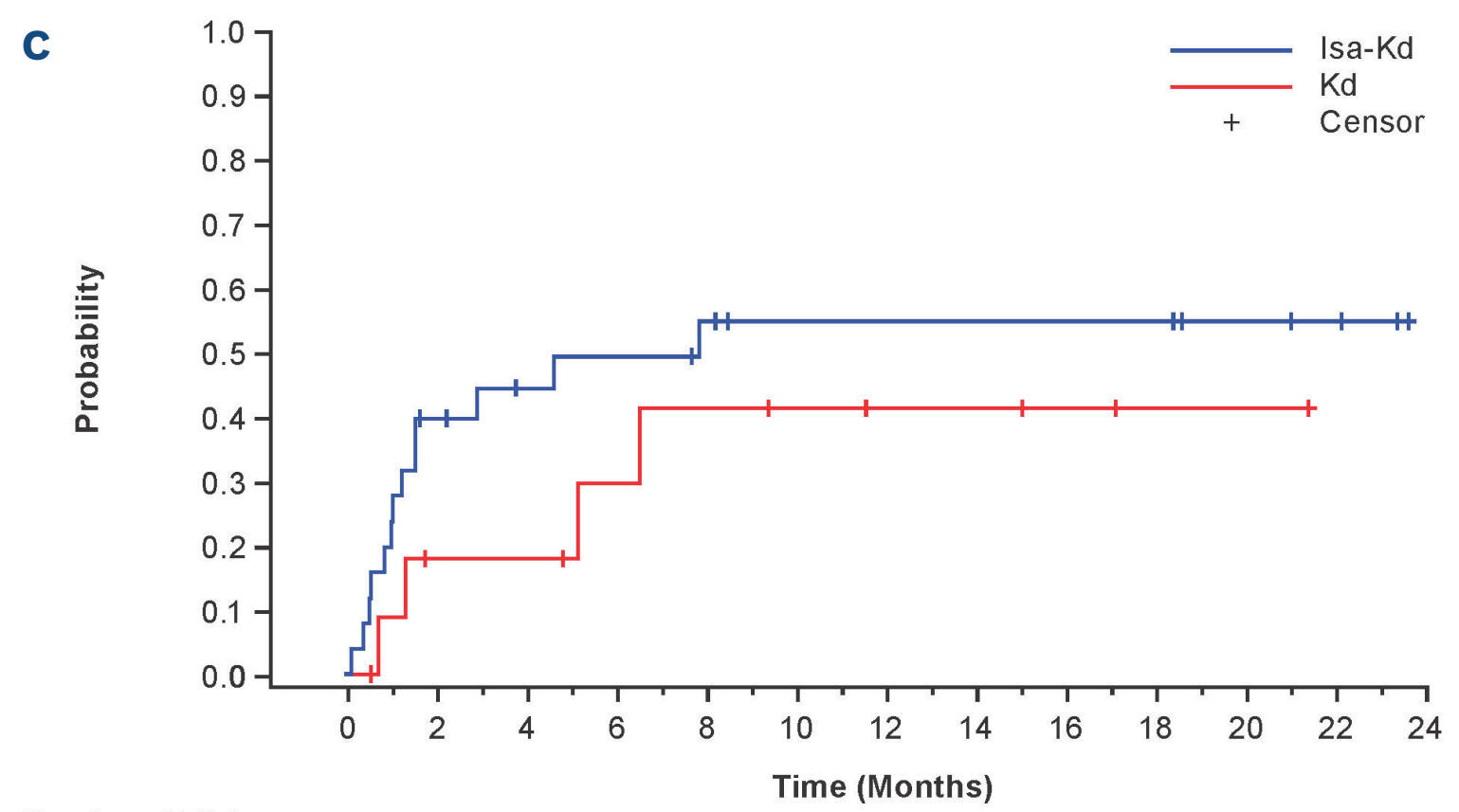

Number at Risk

$\begin{array}{rcccccc}\text { Isa-Kd } & 25 & 14 & 11 & 10 & 6 & 6 \\ \text { Kd } & 13 & 8 & 8 & 6 & 3 & 1\end{array}$

Figure 3. Renal response in the isatuximab (Isa) carfilzomib (K) dexamethasone (d) (IsaKd) compared with Kd arms. (A) Complete and durable $(\geq 60$ days) renal responses in patients with eGFR $<50$ $\mathrm{mL} / \mathrm{min} / 1.73 \mathrm{~m}^{2}$ at baseline and minor renal responses in patients with eGFR $\geq 15$ and $<30$ $\mathrm{mL} / \mathrm{min} / 1.73 \mathrm{~m}^{2}$ at baseline, (ITT population). (B) Time to first renal response and (C) time to first complete renal response in patients with eGFR $<50 \mathrm{~mL} / \mathrm{min} / 1.73 \mathrm{~m}^{2}$ at baseline. CrR: complete renal response; d: dexamethasone; eGFR: estimated glomerular filtration rate; Isa: isatuximab; ITT: intent to treat; $\mathrm{K}$ : carfilzomib; rR: renal response. 
Table 2. Overall extent of exposure in patients with and without renal impairment in the isatuximab (Isa) carfilzomib (K) dexamethasone (d) (Isa-Kd) and Kd arms - safety population

\begin{tabular}{|c|c|c|c|c|}
\hline \multirow{2}{*}{ Exposure } & \multicolumn{2}{|c|}{$\begin{array}{c}\text { eGFR }<60 \mathrm{~mL} / \mathrm{min} / 1.73 \mathrm{~m}^{2} \\
(n=61)\end{array}$} & \multicolumn{2}{|c|}{$\begin{array}{c}\text { eGFR } \geq 60 \mathrm{~mL} / \mathrm{min} / 1.73 \mathrm{~m}^{2} \\
(n=212)\end{array}$} \\
\hline & Isa-Kd $(n=43)$ & $K d(n=18)$ & Isa-Kd $(n=120)$ & $\mathrm{Kd}(\mathrm{n}=92)$ \\
\hline $\begin{array}{l}\text { Median number of cycles } \\
\text { started, (range) }\end{array}$ & $20(2-25)$ & $9(1-24)$ & $19(1-27)$ & $17(1-28)$ \\
\hline $\begin{array}{l}\text { Median treatment } \\
\text { duration, weeks (range) }\end{array}$ & $81.0(6-104)$ & $35.7(1-97)$ & $78.6(1-111)$ & $68.5(4-114)$ \\
\hline \multicolumn{5}{|l|}{$\begin{array}{l}\text { Median relative dose } \\
\text { intensity, \% (range) }\end{array}$} \\
\hline Isatuximab & $94.3(79.2-105.0)$ & - & $94.3(66.7-108.2)$ & - \\
\hline Carfilzomib & $93.1(47.7-108.7)$ & $84.6(44.7-100.2)$ & $90.1(18.2-107.5)$ & $91.4(41.8-108.6)$ \\
\hline Dexamethasone & $85.8(26.8-101.1)$ & $90.2(31.2-100.0)$ & $85.4(24.5-100.2)$ & $88.1(27.4-101.1)$ \\
\hline
\end{tabular}

d: dexamethasone; eGFR: estimated glomerular filtration rate; Isa: isatuximab; K: carfilzomib; RI: renal impairment.

Table 3. Safety summary in patients with and without renal impairment in the isatuximab (Isa) carfilzomib (K) dexamethasone (d) (Isa-Kd) and Kd arms - safety population

\begin{tabular}{|c|c|c|c|c|}
\hline \multirow{2}{*}{ n (\%) } & \multicolumn{2}{|c|}{$\begin{array}{c}\text { eGFR }<60 \mathrm{~mL} / \mathrm{min} / 1.73 \mathrm{~m}^{2} \\
(\mathrm{n}=61)\end{array}$} & \multicolumn{2}{|c|}{$\begin{array}{c}\text { eGFR } \geq 60 \mathrm{~mL} / \mathrm{min} / 1.73 \mathrm{~m}^{2} \\
(n=212)\end{array}$} \\
\hline & $\begin{array}{l}\text { Isa-Kd } \\
(n=43)\end{array}$ & $\begin{array}{c}\text { Kd } \\
(n=18)\end{array}$ & $\begin{array}{l}\text { Isa-Kd } \\
(n=120)\end{array}$ & $\begin{array}{c}\text { Kd } \\
(n=92)\end{array}$ \\
\hline Patients with any TEAE & $42(97.7)$ & $18(100)$ & $116(96.7)$ & $87(94.6)$ \\
\hline $\begin{array}{l}\text { Patients with any grade } \geq 3 \\
\text { TEAE }\end{array}$ & $34(79.1)$ & $14(77.8)$ & $93(77.5)$ & $60(65.2)$ \\
\hline $\begin{array}{l}\text { Patients with any grade } 5 \\
\text { TEAE }^{a}\end{array}$ & 0 & $2(11.1)$ & $5(4.2)$ & $1(1.1)$ \\
\hline Patients with any serious TEAE & $27(62.8)$ & $14(77.8)$ & $71(59.2)$ & $50(54.3)$ \\
\hline $\begin{array}{l}\text { Patients with any TEAE } \\
\text { leading to definitive } \\
\text { discontinuation }\end{array}$ & $3(7.0)$ & $5(27.8)$ & $12(10.0)$ & $9(9.8)$ \\
\hline
\end{tabular}

aTEAE with fatal outcome during the treatment period. d: dexamethasone; eGFR: estimated glomerular filtration rate; Isa: isatuximab; K: carfilzomib; RI: renal impairment; TEAE: treatment-emergent adverse event.

tients. In patients with RI, treatment with Isa-Kd did not increase the incidence of TEAE with fatal outcome during treatment (Isa-Kd, 0\% vs. Kd, 11.1\% [2/18]) nor of TEAE leading to treatment discontinuation (Isa-Kd, $7.0 \%$ vs. Kd, $27.8 \%$, Table 3).

TEAE occurring in $\geq 15 \%$ of patients treated with Isa-Kd are shown in Table 4, by renal function group and treatment arm. In patients with RI, the most common TEAE with Isa$\mathrm{Kd}$ versus $\mathrm{Kd}$ were diarrhea (41.9\% vs. 22.2\%), upper respiratory tract infection (39.5\% vs. 27.8\%), hypertension (34.9\% vs. $27.8 \%$ ), and fatigue (34.9\% vs. $22.2 \%$ ). The most common TEAE with Isa-Kd versus $\mathrm{Kd}$ in patients without RI were hypertension (40.0\% vs. 32.6\%), upper respiratory tract infections (39.2\% vs. $26.1 \%$ ), and diarrhea (36.7\% vs. $31.5 \%)$. Infusion reactions were observed in $37.2 \%$ of Isa$\mathrm{Kd}$ versus $5.6 \%$ of $\mathrm{Kd}$ patients with $\mathrm{RI}$ and $45.8 \%$ of Isa-Kd versus $3.3 \%$ of $\mathrm{Kd}$ patients without $\mathrm{RI}$, but no grade $\geq 3$ infusion reactions were reported. Hypertension was the most common grade $\geq 3$ TEAE independently of renal function: $20.9 \%$ with Isa-Kd versus $22.2 \%$ with $\mathrm{Kd}$ in patients with $\mathrm{RI}$ and $20.8 \%$ with Isa-Kd versus $18.5 \%$ with $\mathrm{Kd}$ in patients without RI (Table 4).

Carfilzomib has been reported to cause cardiac complications. ${ }^{35}$ Cardiac failure (by standardized MedDRA query) was observed in $11.6 \%$ (2.3\% grade $\geq 3$ ) of Isa-Kd patients with $\mathrm{RI}$ versus $5.6 \%$ (5.6\% grade $\geq 3$ ) of $\mathrm{Kd}$ patients with RI. In patients without RI, cardiac failure was observed in $5.8 \%(4.2 \%$ grade $\geq 3)$ of Isa-Kd patients versus $6.5 \%$ (3.3\% grade $\geq 3$ ) of $\mathrm{Kd}$ patients.

The most common hematologic abnormalities based on laboratory results in treated patients with RI were anemia (all patients in both arms) and thrombocytopenia 
(93.0\% with Isa-Kd versus $83.3 \%$ with $\mathrm{Kd}$, Table 5 ). In patients without $\mathrm{RI}$, incidence of anemia was $99.2 \%$ with Isa-Kd versus $100 \%$ with $\mathrm{Kd}$, whereas incidence of thrombocytopenia was $94.2 \%$ with Isa-Kd versus $89.1 \%$ with $\mathrm{Kd}$.
Importantly, the incidence of grade 3-4 anemia and thrombocytopenia were comparable in all subgroups, while the incidence of grade 3 neutropenia was higher in the Isa-Kd arm in patients with or without RI (Table 5).

Table 4. Treatment-emergent adverse events occurring in $\geq 15 \%$ of patients treated with isatuximab (Isa) carfilzomib (K) dexamethasone (d) (Isa-Kd), according to the renal impairment status - safety population.

\begin{tabular}{|c|c|c|c|c|c|c|c|c|}
\hline \multirow{3}{*}{$\begin{array}{l}\text { Most common TEAE (in } \geq 15 \% \\
\text { of patients treated } \\
\text { with Isa-Kd, worst grade) } \\
\text { by preferred term, } n(\%)\end{array}$} & \multicolumn{4}{|c|}{ eGFR $<60 \mathrm{~mL} / \mathrm{min} / 1.73 \mathrm{~m}^{2}(\mathrm{n}=61)$} & \multicolumn{4}{|c|}{ eGFR $\geq 60 \mathrm{~mL} / \mathrm{min} / 1.73 \mathrm{~m}^{2}(n=212)$} \\
\hline & \multicolumn{2}{|c|}{ Isa-Kd $(n=43)$} & \multicolumn{2}{|c|}{$K d(n=18)$} & \multicolumn{2}{|c|}{ Isa-Kd $(n=120)$} & \multicolumn{2}{|c|}{$K d(n=92)$} \\
\hline & Any grade & Grade $\geq 3$ & Any grade & Grade $\geq 3$ & Any grade & Grade $\geq 3$ & Any grade & Grade $\geq 3$ \\
\hline Diarrhea & $18(41.9)$ & 0 & $4(22.2)$ & $1(5.6)$ & $44(36.7)$ & $5(4.2)$ & $29(31.5)$ & $1(1.1)$ \\
\hline Upper respiratory tract infection & $17(39.5)$ & 0 & $5(27.8)$ & $1(5.6)$ & $47(39.2)$ & $6(5.0)$ & $24(26.1)$ & $1(1.1)$ \\
\hline Infusion reaction & $16(37.2)$ & 0 & $1(5.6)$ & 0 & $55(45.8)$ & 0 & $3(3.3)$ & 0 \\
\hline Hypertensiona & $15(34.9)$ & $9(20.9)$ & $5(27.8)$ & $4(22.2)$ & $48(40.0)$ & $25(20.8)$ & $30(32.6)$ & $17(18.5)$ \\
\hline Fatigue & $15(34.9)$ & $4(9.3)$ & $4(22.2)$ & $1(5.6)$ & $35(29.2)$ & $2(1.7)$ & $19(20.7)$ & 0 \\
\hline Dyspnea & $14(32.6)$ & $1(2.3)$ & $2(11.1)$ & 0 & $31(25.8)$ & $8(6.7)$ & $24(26.1)$ & $1(1.1)$ \\
\hline Back pain & $13(30.2)$ & $1(2.3)$ & $2(11.1)$ & 0 & $24(20.0)$ & $2(1.7)$ & $19(20.7)$ & $1(1.1)$ \\
\hline Cough & $10(23.3)$ & 0 & $1(5.6)$ & 0 & $22(18.3)$ & 0 & $16(17.4)$ & 0 \\
\hline Pneumonia & $8(18.6)$ & $5(11.6)$ & $5(27.8)$ & $4(22.2)$ & $31(25.8)$ & $22(18.3)$ & $18(19.6)$ & $11(12.0)$ \\
\hline Bronchitis & $7(16.3)$ & $1(2.3)$ & $1(5.6)$ & 0 & $25(20.8)$ & $3(2.5)$ & $10(10.9)$ & $1(1.1)$ \\
\hline Headache & $7(16.3)$ & 0 & $1(5.6)$ & $1(5.6)$ & $19(15.8)$ & 0 & $19(20.7)$ & 0 \\
\hline Nausea & $7(16.3)$ & 0 & $3(16.7)$ & 0 & $19(15.8)$ & 0 & $16(17.4)$ & 0 \\
\hline Asthenia & $7(16.3)$ & 0 & $3(16.7)$ & $2(11.1)$ & $19(15.8)$ & $3(2.5)$ & 15 (16.3) & $2(2.2)$ \\
\hline Nasopharyngitis & $7(16.3)$ & 0 & $16(13.3)$ & 0 & $7(16.3)$ & 0 & $16(13.3)$ & 0 \\
\hline Edema peripheral & $7(16.3)$ & 0 & $2(11.1)$ & 0 & $14(11.7)$ & $1(0.8)$ & $17(18.5)$ & 0 \\
\hline Fall & $7(16.3)$ & $2(4.7)$ & 0 & 0 & $12(10.0)$ & $1(0.8)$ & $10(10.9)$ & 0 \\
\hline Insomnia & $5(11.6)$ & $1(2.3)$ & $3(16.7)$ & $1(5.6)$ & $32(26.7)$ & $6(5.0)$ & $23(25.0)$ & $2(2.2)$ \\
\hline Vomiting & $5(11.6)$ & 0 & $3(16.7)$ & 0 & $18(15.0)$ & $2(1.7)$ & $8(8.7)$ & $1(1.1)$ \\
\hline
\end{tabular}

'Both new hypertension and worsening hypertension were included in the preferred term 'hypertension'; hypertension in medical history was $41.0 \%$ in Isa-Kd vs. $18 \%$ in $\mathrm{Kd}$ in patients with RI and $24.7 \%$ in Isa-Kd vs. $12.1 \%$ in $\mathrm{Kd}$ in patients without RI. d: dexamethasone; eGFR: estimated glomerular filtration rate; Isa: isatuximab; K: carfilzomib; RI: renal impairment; TEAE: treatment-emergent adverse event.

Table 5. Hematologic abnormalities determined by laboratory analysis in patients with and without renal impairment in the isatuximab (Isa) carfilzomib (K) dexamethasone (d) (Isa-Kd) and Kd arms - safety population.

\begin{tabular}{|c|c|c|c|c|c|c|c|c|c|c|c|c|}
\hline \multirow{3}{*}{$\begin{array}{l}\text { Hematologic } \\
\text { laboratory } \\
\text { abnormalities }{ }^{\mathrm{a}} \text {, } \\
\text { n (\%) }\end{array}$} & \multicolumn{6}{|c|}{ eGFR $<60 \mathrm{~mL} / \mathrm{min} / 1.73 \mathrm{~m}^{2}(\mathrm{n}=61)$} & \multicolumn{6}{|c|}{ eGFR $\geq 60 \mathrm{~mL} / \mathrm{min} / 1.73 \mathrm{~m}^{2}(\mathrm{n}=212)$} \\
\hline & \multicolumn{3}{|c|}{ Isa-Kd ( $n=43)$} & \multicolumn{3}{|c|}{$K d(n=18)$} & \multicolumn{3}{|c|}{ Isa-Kd $(n=120)$} & \multicolumn{3}{|c|}{$K d(n=92)$} \\
\hline & $\begin{array}{c}\text { Any } \\
\text { grade }\end{array}$ & $\begin{array}{c}\text { Grade } \\
3\end{array}$ & $\begin{array}{c}\text { Grade } \\
4\end{array}$ & $\begin{array}{c}\text { Any } \\
\text { grade }\end{array}$ & $\begin{array}{c}\text { Grade } \\
3\end{array}$ & $\begin{array}{c}\text { Grade } \\
4\end{array}$ & $\begin{array}{c}\text { Any } \\
\text { grade }\end{array}$ & $\begin{array}{c}\text { Grade } \\
3\end{array}$ & $\begin{array}{c}\text { Grade } \\
4\end{array}$ & $\begin{array}{c}\text { Any } \\
\text { grade }\end{array}$ & $\begin{array}{c}\text { Grade } \\
3\end{array}$ & $\begin{array}{c}\text { Grade } \\
4\end{array}$ \\
\hline Anemia & $43(100)$ & $11(25.6)$ & 0 & $18(100)$ & $5(27.8)$ & 0 & $119(99.2)$ & 23 (19.2) & 0 & $92(100)$ & $14(15.2)$ & 0 \\
\hline Thrombocytopenia & $40(93.0)$ & $9(20.9)$ & $8(18.6)$ & $15(83.3)$ & $6(33.3)$ & $2(11.1)$ & $113(94.2)$ & $19(15.8)$ & $10(8.3)$ & $82(89.1)$ & $12(13.0)$ & $6(6.5)$ \\
\hline Neutropenia & $24(55.8)$ & $6(14.0)$ & $1(2.3)$ & 7 (38.9) & 0 & 0 & $67(55.8)$ & $23(19.2)$ & $2(1.7)$ & $43(46.7)$ & $8(8.7)$ & 0 \\
\hline
\end{tabular}




\section{Discussion}

In MM patients, RI is considered a poor prognostic factor, being associated with earlier mortality and worse OS. 5,22,36 As such, there is a critical need for anti-MM therapies that also improve renal function. This prespecified subgroup analysis of the phase III IKEMA study demonstrated that Isa$\mathrm{Kd}$ is efficacious and has a manageable safety profile in patients with RI.

The risk of disease progression or death in patients with RI was $73 \%$ lower in the Isa-Kd arm, indicated by the very low HR (HR: 0.27; 95\% Cl: 0.11-0.66). This result is consistent with the PFS benefit of Isa-Kd observed in the overall IKEMA study population (HR: 0.53; 99\% Cl: $0.32-0.89)^{28}$ and in patients without RI (HR: 0.63; 95\% Cl: 0.39-1.00). In IKEMA, median PFS observed among patients with RI (eGFR <60 $\mathrm{mL} / \mathrm{min} / 1.73 \mathrm{~m}^{2}$ ) receiving $\mathrm{Kd}$ (13.4 months) was similar to results of the $\mathrm{Kd}$ arm in the ENDEAVOR subgroup analysis, with a median PFS of 14.9 months in patients with creatinine clearance $\geq 15$ to $<50 \mathrm{~mL} / \mathrm{min}$ (severe/moderate $\mathrm{RI}$ ). ${ }^{9}$ Consistent with the PFS results, duration of study treatment exposure was similar in Isa-Kd patients with or without RI (81 and 78.6 months, respectively), whereas it was shorter in Kd patients with RI versus those without RI (35.7 vs. 68.5 months, respectively).

The ORR was greater with Isa-Kd than Kd in patients with RI (93.1\% vs. 61.1\%), whereas patients without RI showed similar ORR in the two study arms (83.6\% vs. 89.2\%), consistent with the overall IKEMA study population (86.6\% Isa$\mathrm{Kd}$ vs. $82.9 \% \mathrm{Kd}) .{ }^{28}$ Of note, depth of response was superior with Isa-Kd versus $\mathrm{Kd}$ independently of $\mathrm{RI}$ status, with respect to $C R$ rate $(41.9 \%$ Isa-Kd vs. $22.2 \% \mathrm{Kd}$ in patients with $\mathrm{RI}$; $40.2 \%$ Isa-Kd versus $30.1 \% \mathrm{Kd}$ in patients without $\mathrm{RI}$ ), $\geq$ VGPR rate $(79.1 \%$ Isa-Kd vs. $44.4 \% \mathrm{Kd}$ in patients with RI; $71.3 \%$ Isa-Kd vs. $59.1 \% \mathrm{Kd}$ in patients without RI), and MRD negativity rate $(30.2 \%$ Isa-Kd vs. $11.1 \% \mathrm{Kd}$ in patients with $\mathrm{RI}$; 29.5\% Isa-Kd vs. $14.0 \% \mathrm{Kd}$ in patients without RI).

Remarkably, compared with $\mathrm{Kd}$, Isa-Kd increased the proportion of patients with RI who achieved both complete (52.0\% Isa-Kd vs. $30.8 \% \mathrm{Kd}$ ) and durable ( $\geq 60$ days; $32.0 \%$ Isa-Kd vs. $7.7 \% \mathrm{Kd}$ ) renal responses, and decreased time to first (1.5 months Isa-Kd vs. 6.5 months $\mathrm{Kd}$ ) and to complete (7.8 months Isa-Kd vs. NC Kd) renal response, suggesting that Isa-Kd allows the achievement of sustainable reversal of RI. Similarly, compared with Kd, fewer patients in the Isa$\mathrm{Kd}$ arm experienced worsening of renal function or progression to end-stage RI.

The addition of Isa to Kd was associated with a manageable safety profile in MM patients with and without RI. Among patients with RI, there was a similar incidence of patients with grade $\geq 3$ TEAE between the two arms (79.1\% Isa-Kd vs. $77.8 \% \mathrm{Kd}$ ), whereas this incidence was higher in patients without RI (77.5\% Isa-Kd vs. $65.2 \%$ Kd). Furthermore, in patients with RI there was a similar incidence of patients with serious TEAE or TEAE leading to death during study treatment or treatment discontinuation. The higher treatment exposure observed with Isa-Kd versus Kd might have contributed to the higher incidence of grade $\geq 3$ TEAE in patients without RI. Cardiac failure in the overall population was similar between study arms (7.3\% all grades and $4.0 \%$ grade $\geq 3$ in Isa-Kd versus $6.6 \%$ all grades and $4.1 \%$ grade $\geq 3$ in $\mathrm{Kd}$ ), but incidence of any-grade cardiac failure was higher in patients with RI in Isa-Kd (11.6\% vs. 5.6\%). This can be related to a longer treatment exposure in Isa-Kd (median number of cycles was 20 in Isa-Kd vs. 9 in Kd) and higher carfilzomib relative dose intensity in Isa-Kd (93.1\% vs. 84.6\%). This difference in incidence disappeared for grade $\geq 3$ events.

The most common TEAE in patients with RI treated with Isa-Kd versus Kd were diarrhea, upper respiratory tract infection, hypertension, and fatigue with similar frequency observed in the overall IKEMA population. ${ }^{28}$ There was no increased incidence of infusion reactions in the $\mathrm{RI}(37.2 \%$ Isa-Kd vs. $5.6 \% \mathrm{Kd}$ ) compared with non-RI (45.8\% Isa-Kd vs. $3.3 \% \mathrm{Kd}$ ) subgroups.

There are few reports in the literature analyzing the efficacy and toxicity of anti-CD38 monoclonal antibodies in patients with RI. The results of this IKEMA subgroup analysis reinforce the findings of the ICARIA-MM RI subgroup analysis, which showed that addition of Isa to Pd also improved clinical outcomes in patients with RI. ${ }^{27}$ Median PFS was 9.5 months with Isa-Pd versus 3.7 months with Pd (HR: 0.50; 95\% Cl: 0.30-0.85) for patients with RI. Isa-Pd also showed greater depth of response in patients with RI, with a $56 \%$ ORR with Isa-Pd versus $25 \%$ with $\mathrm{Pd}$. Complete renal response rates were achieved in 23 of 32 (71.9\%) patients treated with Isa-Pd and eight of 21 (38.1\%) treated with Pd; these were durable in ten of 32 (31.3\%) and four of 21 (19.0\%) of patients treated with Isa-Pd versus $\mathrm{Pd}$, respectively. ${ }^{27}$

Data about efficacy and safety of daratumumab, a different CD38 monoclonal antibody, in patients with RI are limited. A few, isolated case reports with single dialysis-dependent patients have been published. ${ }^{37-40}$ Results from a retrospective, multicenter, open-label study designed to evaluate safety and efficacy of daratumumab in RRMM patients with end-stage $\mathrm{RI}$ requiring hemodialysis $(n=15)$ reported a median PFS of 8.7 months, OS of 12.2 months, and ORR of $40 \% .{ }^{41}$ The most common grade 3-4 hematologic AE included thrombocytopenia $(n=5)$, anemia $(n=4)$, and neutropenia $(n=4)$. Infusion reactions $(n=6)$ were the most frequent non-hematologic AE. ${ }^{41}$ Results of an interim analysis of the phase II DARE study, a multicenter, single-arm, open-label study in RRMM patients with severe RI (eGFR $<30 \mathrm{~mL} / \mathrm{min} / 1.73 \mathrm{~m}^{2}$ ) or in need of hemodialysis were reported recently. ${ }^{42}$ Eligible patients had received $\geq 2$ prior treatment lines (including bortezomib- and lenalidomidebased regimens) and presented with ECOG PS score $\leq 2$. At the cut-off date, 35 patients treated with daratumumab and dexamethasone showed a 12-month PFS probability of $50 \%$, 
an ORR of $45.7 \%$, and a renal response rate of $17.1 \%$. The most common grade 3-4 AE were anemia (17.1\%) and hyperglycemia (8.6\%). A total of $48.6 \%$ of patients had $\geq 1$ grade 3-4 AE and $25.7 \%$ of patients experienced $\geq 1$ serious $A E{ }^{42}$ Limitations of this IKEMA subgroup analysis include: (i) less than $3 \%$ of patients in each arm had severe RI, so the results presented here are mainly applicable to patients with moderate RI, and (ii) RI status at baseline was not a stratification factor, likely resulting in small differences in subgroup size between treatment arms.

In summary, addition of Isa to Kd improved PFS and depth of response in patients with relapsed $\mathrm{MM}$ and $\mathrm{RI}$, with a manageable safety profile, consistent with the benefit observed in the overall IKEMA study population. More patients treated with Isa-Kd showed reversal of RI and durable renal responses compared with those treated with $\mathrm{Kd}$. Based on these findings, Isa-Kd represents a valuable addition to the therapies used to treat patients with $\mathrm{MM}$-related renal dysfunction.

\section{Disclosures}

MC is part of the speaker's bureau of Amgen, Janssen, and Sanofi. TM has received research funding from Amgen, Janssen, and Sanofi; consults for GSK. PM has received honoraria from Amgen, Celgene, Janssen, Novartis, and Takeda; has a consulting or advisory role at Amgen, Celgene, Janssen, Novartis, and Takeda. RB has received research funding from AbbVie, Acerta Pharma, Alexion, Amgen, Bayer, Boehringer Ingelheim, Bristol Myers Squibb, Celgene, CSL Behring, Daiichi Sankyo, Janssen-Cilag, Morphosys AG, Pfizer, Rigel Pharmaceuticals, Roche, Sanofi, and Takeda; has received honoraria from Bayer; has a consulting or advisory role at Janssen-Cilag, Roche; is part of the speaker's bureau of Bayer. LP, C-KM, MRS, and MT have no conflicts of interest to disclose. $X L$ has received honoraria from AbbVie, Amgen, Bristol Myers Squibb, Carsgen Therapeutics Ltd, Celgene, Gilead Sciences, Janssen-Cilag, Karyopharm Therapeutics, Merck, Mundipharma, Novartis, Oncopeptides, Pierre Fabre, Roche, Sanofi, and Takeda; has received non-financial support from Takeda. MM has received research funding from Adaptive, Amgen, Bristol Myers Squibb, Celgene, GlaxoSmithKline, Janssen, Jazz,
Novartis, Sanofi, Stemline Therapeutics, and Takeda; has received honoraria from Adaptive, Amgen, Bristol Myers Squibb, Celgene, GlaxoSmithKline, Janssen, Jazz, Novartis, Sanofi, Stemline Therapeutics, and Takeda; has received non-financial support from Takeda; has a consulting or advisory role at Adaptive, Amgen, Bristol Myers Squibb, Celgene, GlaxoSmithKline, Janssen, Jazz, Novartis, Sanofi, Stemline Therapeutics, and Takeda. RLB has a consulting or advisory role at Celgene/Bristol Myers Squibb, Janssen, Amgen, Sanofi, and Takeda; has received research funding from Celgene/Bristol Myers Squibb. M-LR and SS are employed by Sanofi; may hold stock and/or stock options in the company. LM has a consulting or advisory role at Aixial (contracted by Sanofi). MD has a consulting or advisory role at Amgen, Bristol Myers Squibb, Celgene, Janssen, and Takeda.

\section{Contributions}

IKEMA Study Steering Committee members (TM, PM, MD) and employees of Sanofi (M-LR, SS) contributed to the conception/design of this study. All authors contributed to the provision of study material, data collection and analysis, as well as development/final approval of the manuscript.

\section{Acknowledgments}

The IKEMA study was sponsored by Sanofi. We thank the participating patients and their caregivers, and the study centers and investigators for their contributions to the study. Medical writing support was provided by C. Semighini Grubor, PhD, and S. Mariani, MD, PhD, of Elevate Medical Affairs, contracted by Sanofi Genzyme, for publication support services.

\section{Data sharing statement}

Qualified researchers can request access to patient-level data and related study documents including the clinical study report, study protocol with any amendments, blank case report forms, statistical analysis plan, and dataset specifications. Patient-level data will be anonymized, and study documents will be redacted to protect the privacy of trial participants. Further details on Sanofi's data-sharing criteria, eligible studies, and process for requesting access are at: https://www.clinicalstudydatarequest.com.

\section{References}

1. Clark AD, Shetty A, Soutar R. Renal failure and multiple myeloma: pathogenesis and treatment of renal failure and management of underlying myeloma. Blood Rev. 1999;13(2):7990.

2. Eleutherakis-Papaiakovou V, Bamias A, Gika D, et al. Renal failure in multiple myeloma: incidence, correlations, and prognostic significance. Leuk Lymphoma. 2007;48(2):337-341.

3. San-Miguel JF, Richardson PG, Sonneveld P, et al. Efficacy and safety of bortezomib in patients with renal impairment: results from the APEX phase 3 study. Leukemia. 2008;22(4):842-849.

4. Yadav P, Cook M, Cockwell P. Current trends of renal impairment in multiple myeloma. Kidney Dis (Basel). 2016;1(4):241-257.

5. Fotiou D, Dimopoulos MA, Kastritis E. Managing renal complications in multiple myeloma. Expert Rev Hematol. 2016;9(9):839-850.

6. Dimopoulos MA, Terpos E, Chanan-Khan A, et al. Renal impairment in patients with multiple myeloma: a consensus statement on behalf of the International Myeloma Working 
Group. J Clin Oncol. 2010;28(33):4976-4984.

7. Dimopoulos MA, Sonneveld P, Leung N, et al. International Myeloma Working Group recommendations for the diagnosis and management of myeloma-related renal impairment. J Clin Oncol. 2016;34(13):1544-1557.

8. Dimopoulos MA, Moreau P, Palumbo A, et al. Carfilzomib and dexamethasone versus bortezomib and dexamethasone for patients with relapsed or refractory multiple myeloma (ENDEAVOR): a randomised, phase 3, open-label, multicentre study. Lancet Oncol. 2016;17(1):27-38.

9. Dimopoulos M, Siegel D, White DJ, et al. Carfilzomib vs bortezomib in patients with multiple myeloma and renal failure: a subgroup analysis of ENDEAVOR. Blood. 2019;133(2):147-155.

10. Dimopoulos MA, Richardson PG, Schlag R, et al. VMP (bortezomib, melphalan, and prednisone) is active and well tolerated in newly diagnosed patients with multiple myeloma with moderately impaired renal function, and results in reversal of renal impairment: cohort analysis of the phase III VISTA study. J Clin Oncol. 2009;27(36):6086-6093.

11. Dimopoulos MA, Roussou M, Gavriatopoulou M, et al. Reversibility of renal impairment in patients with multiple myeloma treated with bortezomib-based regimens: identification of predictive factors. Clin Lymphoma Myeloma. 2009;9(4):302-306.

12. Dimopoulos MA, Roussou M, Gavriatopoulou M, et al. Bortezomib-based triplets are associated with a high probability of dialysis independence and rapid renal recovery in newly diagnosed myeloma patients with severe renal failure or those requiring dialysis. Am J Hematol. 2016;91(5):499-502.

13. Weisel KC, Dimopoulos MA, Moreau P, et al. Analysis of renal impairment in MM-003, a phase III study of pomalidomide + low-dose dexamethasone versus high-dose dexamethasone in refractory or relapsed and refractory multiple myeloma. Haematologica. 2016;101(7):872-878.

14. Gavriatopoulou M, Terpos E, Dimopoulos MA. IMiDs for myeloma induced renal impairment. Oncotarget. 2018;9(84):35476-35477.

15. Dimopoulos M, Weisel K, van de Donk N, et al. Pomalidomide plus low-dose dexamethasone in patients with relapsed/refractory multiple myeloma and renal impairment: results from a Phase II trial. J Clin Oncol. 2018;36(20):20352043.

16. Siegel DS, Weisel KC, Dimopoulos MA, et al. Pomalidomide plus low-dose dexamethasone in patients with relapsed/refractory multiple myeloma and moderate renal impairment: a pooled analysis of three clinical trials. Leuk Lymphoma. 2016;57(12):2833-2838.

17. Dimopoulos M, Alegre A, Stadtmauer EA, et al. The efficacy and safety of lenalidomide plus dexamethasone in relapsed and/or refractory multiple myeloma patients with impaired renal function. Cancer. 2010;116(16):3807-3814.

18. Dimopoulos MA, Cheung MC, Roussel M, et al. Impact of renal impairment on outcomes with lenalidomide and dexamethasone treatment in the FIRST trial, a randomized, open-label phase 3 trial in transplant-ineligible patients with multiple myeloma. Haematologica. 2016;101(3):363-370.

19. Dimopoulos MA, Christoulas D, Roussou M, et al. Lenalidomide and dexamethasone for the treatment of refractory/relapsed multiple myeloma: dosing of lenalidomide according to renal function and effect on renal impairment. Eur $\mathrm{J}$ Haematol. 2010;85(1):1-5.

20. Dimopoulos MA, Terpos E, Goldschmidt H, Alegre A, Mark T, Niesvizky R. Treatment with lenalidomide and dexamethasone in patients with multiple myeloma and renal impairment. Cancer Treat Rev. 2012;38(8):1012-1019.
21. Dimopoulos MA, Roussou M, Gkotzamanidou M, et al. The role of novel agents on the reversibility of renal impairment in newly diagnosed symptomatic patients with multiple myeloma. Leukemia. 2013;27(2):423-429.

22. Park S, Han B, Kim K, et al. Renal insufficiency in newlydiagnosed multiple myeloma: analysis according to International Myeloma Working Group consensus statement. Anticancer Res. 2014;34(8):4299-4306.

23. Kyprolis $®$ (carfilzomib) for injection, for intravenous use [prescribing information], Amgen, Thousand Oaks, CA (March 2021).

24. Sarclisa ${ }^{\circledR}$ (isatuximab-irfc) injection, for intravenous use [prescribing information], Sanofi, Bridgewater, NJ (March 2021).

25. European Medicines Agency. Sarclisa, INN-Ixatuximab. Summary of product characteristics. 2021.

https://www.ema.europa.eu/en/documents/productinformation/sarclisa-epar-product-information_en.pdf. Accessed May 13, 2021.

26. Attal M, Richardson PG, Rajkumar SV, San-Miguel J, Beksac M, Spicka I; ICARIA-MM study group. Isatuximab plus pomalidomide and low-dose dexamethasone versus pomalidomide and low-dose dexamethasone in patients with relapsed and refractory multiple myeloma (ICARIA-MM): a randomised, multicentre, open-label, phase 3 study. Lancet. 2019;394(10214):2096-2107.

27. Dimopoulos MA, Leleu X, Moreau P, et al. Isatuximab plus pomalidomide and dexamethasone in relapsed/refractory multiple myeloma patients with renal impairment: ICARIA-MM subgroup analysis. Leukemia. 2021;35(2):562-572.

28. Moreau P, Dimopoulos M-A, Mikhael J, et al. Isatuximab, carfilzomib, and dexamethasone in relapsed multiple myeloma (IKEMA): a multicentre, open-label, randomised phase 3 trial. Lancet. 2021;397(10292):2361-2371.

29. Moreau P, Dimopoulos MA, Yong K, et al. Isatuximab plus carfilzomib/dexamethasone versus carfilzomib/dexamethasone in patients with relapsed/refractory multiple myeloma: IKEMA Phase III study design. Future Oncol. 2020;16(2):4347-4358.

30. Levey AS, Bosch JP, Lewis JB, Greene T, Rogers N, Roth D. A more accurate method to estimate glomerular filtration rate from serum creatinine: a new prediction equation. Modification of Diet in Renal Disease Study Group. Ann Intern Med. 1999;130(6):461-470.

31. Rajkumar SV, Harousseau JL, Durie B, et al. Consensus recommendations for the uniform reporting of clinical trials: report of the International Myeloma Workshop Consensus Panel 1. Blood. 2011;117(18):4691-4695.

32. Kumar S, Paiva B, Anderson KC, et al. International Myeloma Working Group consensus criteria for response and minimal residual disease assessment in multiple myeloma. Lancet Oncol. 2016;17(8):e328-346.

33. Lahuerta JJ, Paiva B, Vidriales MB, et al. Depth of response in multiple myeloma: a pooled analysis of three PETHEMA/GEM clinical trials. J Clin Oncol. 2017;35(25):2900-2910.

34. Paiva B, Puig N, Cedena MT, et al. Measurable residual disease by next-generation flow cytometry in multiple myeloma. J Clin Oncol. 2020;38(8):784-792.

35. Dimopoulos MA, Roussou M, Gavriatopoulou M, et al. Cardiac and renal complications of carfilzomib in patients with multiple myeloma. Blood Adv. 2017;1(7):449-454.

36. Augustson BM, Begum G, Dunn JA, et al. Early mortality after diagnosis of multiple myeloma: analysis of patients entered onto the United kingdom Medical Research Council trials between 1980 and 2002--Medical Research Council Adult Leukaemia Working Party. J Clin Oncol. 2005;23(36):9219-9226. 
37. Rocchi S, Tacchetti P, Pantani L, et al. Safety and efficacy of daratumumab in dialysis-dependent renal failure secondary to multiple myeloma. Haematologica. 2018;103(6):e277-278.

38. Smyth E, Glavey S, Melotti D, et al. Dialysis independence following single-agent daratumumab in refractory myeloma with renal failure. Ir J Med Sci. 2019;188(3):1079-1080.

39. Moore DC, Arnall JR, Janes A, Pineda-Roman M. Dialysis independence following combination daratumumab, thalidomide, bortezomib, cyclophosphamide, and dexamethasone in multiple myeloma with severe renal failure. Clin Lymphoma Myeloma Leuk. 2020;20(7):e395-398.
40. Jeyaraman P, Bhasin A, Dayal N, Pathak S, Naithani R. Daratumumab in dialysis-dependent multiple myeloma. Blood Res. 2020;55(1):65-67.

41. Cejalvo MJ, Legarda M, Abella E, et al. Single-agent daratumumab in patients with relapsed and refractory multiple myeloma requiring dialysis: results of a Spanish retrospective, multicentre study. Br J Haematol. 2020;190(5):e289-292.

42. Kastritis E, Terpos E, Symeonidis A, et al. Daratumumab with dexamethasone in patients with relapsed/refractory multiple myeloma and severe renal impairment: results on efficacy and safety of the Phase 2 Dare study. Blood. 2020;136(Suppl 1):S48-49. 